\title{
Articles
}

\section{Azide/Alkyne Resins for Quick Preparation of 1,4-Disubstituted 1,2,3-Triazoles}

\author{
Uthaiwan Sirion, ${ }^{a}$ Jae Hak Lee, ${ }^{\mathrm{b}}$ Yu Jin Bae, Hee Jun Kim, Byoung Se Lee, ${ }^{\dagger, *}$ and Dae Yoon Chi ${ }^{\dagger+, * *}$ \\ Department of Chemistry, Inha University, Inchon 402-751, Korea \\ ${ }^{\dagger}$ Research Institute of Labeling, FutureChem Co., Ltd, Seoul 138-736, Korea. *E-mail: bslee@futurechem.co.kr \\ ${ }^{\ddagger}$ Department of Chemistry, Sogang University, Seoul 121-742, Korea. *E-mail: dychi@sogang.ac.kr \\ Received October 14, 2009, Accepted November 16, 2009
}

\begin{abstract}
An efficient method for the preparation of 1,4-disubstituted 1,2,3-triazole compounds is described using polymeric quaternary ammonium salts having azide or alkyne functionality to remove unreacted excess starting molecules (azide/alkyne). Copper metal could easily be removed by simple filtration with a short $\mathrm{Na}_{2} \mathrm{SO}_{4} /$ silica cartridge, affording highly regioselective products in high yield and excellent purity without the need for work-up, extraction and chromatographic purification.
\end{abstract}

Key Words: Click chemistry, Azide/alkyne resins, 1,2,3-Triazole, Huisgen 1,3-dipolar cycloaddition reaction

\section{Introduction}

Copper(I)-catalyzed Huisgen 1,3-dipolar cycloaddition reaction, described by the Meldal ${ }^{1}$ and Sharpless ${ }^{2}$ groups, has been used extensively as a powerful tool in various fields of applied chemistry $^{3-5}$ due to its rapid, mild, specific, and air/moisturetolerant reaction. The triazole ring is stable to acid/basic hydrolysis as well as reductive/oxidative conditions, and displays desirable features for use as a drug. ${ }^{7}$ Several bioconjugations have utilized triazole moiety as an isostere of the amide bond. ${ }^{8}$ This heterocyclic ring has received increasing research attention in the fields of synthetic organic chemistry, medicinal chemistry, and material science. The development of new process for rapid purification has become an important challenge from practical, economical, and environmental points of view. Although click reaction is carried out readily with high yield, purification of product still requires high research cost. Generally, one excess starting molecule, copper metal, some additives such as sodium ascorbate, amine bases, ligands, and solvent remain after complete click reaction and require typical purification procedures such as extraction and chromatographical separation, consuming expensive time and cost. Some efforts have been made to achieve efficient purification-free methods. Recently, Girard et al. have reported dimethylaminomethyl polystyrene-supported $\mathrm{Cu}(\mathrm{I})$ for the efficient removal and reuse of the $\mathrm{Cu}(\mathrm{I})$ catalyst. ${ }^{9}$ Similarly, in our previous work we prepared CuI-immobilized ionic polymers as a reusable catalyst for Huisgen 1,3dipolar cycloaddition reaction. ${ }^{10}$ This catalyst showed good catalytic activity up to 10 reuses without significant leaching of $\mathrm{CuI}$ and any loss of yield. In addition, Smith et al. described the successful removal of $\mathrm{CuI}$ and excess azide using a modular flow reactor system comprised of three continuous columns

${ }^{a}$ Present address: Department of Chemistry, Burapha University, Sangsaen, Chonburi 20131, Thailand.

${ }^{b}$ Present address: Department of Chemistry, Sogang University, Seoul 121-742, Korea. containing different polymer resins. ${ }^{11}$

Herein, we report a very simple and expedient method for the rapid preparation of 1,4-disubstituted 1,2,3-triazole using scavenger resins to remove unreacted azide/alkyne after the solution-phase click reaction. Subsequently, copper catalyst is also removed by simple filtration. This work was motivated by the necessary requirements that must be satisfied for potential application to high throughput synthesis of 1,4-disubstituted 1,2,3-triazoles.

\section{Experimental Section}

General methods. All chemicals were purchased from commercial sources and used without further purification. Quaternization of resins and reaction process were carried out using a speed control shaker, BTS-1500/RB-20/RBTC-T/150-T of J-KEM Scientific, Inc. ${ }^{1} \mathrm{H}$ and ${ }^{13} \mathrm{C}$ NMR spectra were recorded using both Varian Gemini-2000 (200 MHz) and Varian UNITY-INOVA 400 (400 MHz). ICP-MS (Inductively Coupled Plasma - Mass Spectrometer) data were obtained to quantify copper metal using ELAN6100/Perkin Elmer. Low-resolution mass spectra were obtained using VK Quattro II GC-MS/MS spectrometer (ESI or EI). Elemental Analyzer (EA), Flash EA1112/Thermo Electron was used to calculate nitrogen content of azide (A) and acetylene (B) resins. High-resolution mass spectra (HRMS) data were recorded at the Korea Basic Science Institute using Jeol-JMS 700. Melting Points were checked with OptiMelt apparatus (Stanford research systems). High performance liquid chromatography (HPLC) was performed using a Varian ProStar, HPLC Condition: (Polaris 5C 18-A, batch: 2620101, SS $250 \mathrm{~mm} \times 4.6 \mathrm{~mm}$ ) at the flow-rate of $1 \mathrm{~mL} / \mathrm{min}$ eluting with $\mathrm{CH}_{3} \mathrm{CN} / \mathrm{H}_{2} \mathrm{O}(45 / 55)$.

Preparation of scavenger resins A and B (Scheme 1). Chloromethyl Merrifield resin $(2.00 \mathrm{~g}, 3.60 \mathrm{mmol}$ of $\mathrm{Cl})$ was placed in a vial pretreated with Sigmacote ${ }^{\circledR}$ and DMA $(25 \mathrm{~mL})$ was added. After the resin was fully swollen, dimethylamino-2- 
propyne ( $768 \mu \mathrm{L}, 7.20 \mathrm{mmol})$ or dimethylamino-1-azidopropane (923 $\mu \mathrm{L}, 7.20 \mathrm{mmol}$ ) was added to the suspension. Using a block shaker equipped with speed and temperature controller, the suspension was well shaken at room temperature for $24 \mathrm{~h}$. The resulting ionic resin was collected into a plastic syringe equipped with polyethylene frit, and counter anion, chloride were exchanged by simple ion exchange process with aqueous solution of sodium tetrafluoroborate $(0.2 \mathrm{M}, 50 \mathrm{~mL})$, this ion exchange procedure was repeated 10 times, and then washed with distilled water, acetone, methanol, THF, and diethyl ether, and dried under reduced pressure to give resin $\mathbf{A}(2.592 \mathrm{~g}, 1.376$ $\mathrm{mmol} / \mathrm{g}$ of acetylene group determined by nitrogen content of elemental analysis) or resin B (2.600 g, $1.249 \mathrm{mmol} / \mathrm{g}$ of azide determined by nitrogen content of elemental analysis).

General procedure for 1,2,3-triazole synthesis (Table 2). Aqueous $\mathrm{CuSO}_{4} \cdot 5 \mathrm{H}_{2} \mathrm{O}(5 \mathrm{~mol} \%, 0.2 \mathrm{M}, 125 \mu \mathrm{L}), \mathrm{Cu}$ powder $(5 \mathrm{~mol} \%, 2 \mathrm{mg})$ and $\mathrm{Et}_{3} \mathrm{~N}(5 \mathrm{~mol} \%, 3.5 \mu \mathrm{L})$ were added into a vial containing $\mathrm{CH}_{3} \mathrm{CN}(1.5 \mathrm{~mL})$, and then azide/alkyne compound $(0.50 \mathrm{mmol})$, alkyne/azide compound $(0.55 \mathrm{mmol})$ were added to the solution. The reaction mixture was shaken using a shaker at room temperature and monitored by TLC. After complete conversion, to the reaction mixture was added resin A or $\mathbf{B}$ (depending on type of excess starting molecule), and the suspension was shaken using a shaker at room temperature until the excess starting molecule disappeared on TLC. The reaction mixture was diluted with ethyl acetate $(3.0 \mathrm{~mL})$ and filtered with a short silica/ $\mathrm{Na}_{2} \mathrm{SO}_{4}$ column, and then washed with ethyl acetate $(5.0 \mathrm{~mL} \times 2)$. The combined filtrate was concentrated under reduced pressure to give the crude product, whose purity was determined by NMR and HPLC data without further purification.

1-Benzyl-4-phenyl-1H-1,2,3-triazole (entry 1, Table 2). CAS No. 108717-96-0; 99\% yield as a white solid: mp $104-105^{\circ} \mathrm{C}$; ${ }^{1} \mathrm{H} \mathrm{NMR}\left(\mathrm{CDCl}_{3}, 200 \mathrm{MHz}\right)$ 7.82-7.77 (m, 2H), 7.66 (s, 1H), 7.43-7.26 (m, 8H), $5.56(\mathrm{~s}, 2 \mathrm{H}) ;{ }^{13} \mathrm{C} \mathrm{NMR}\left(\mathrm{CDCl}_{3}, 50 \mathrm{MHz}\right)$ $\delta 148.3,134.7,130.5,129.1,128.7,128.1,128.0,125.7,119.5$, 54.2. HRMS (FAB) $m / z \mathrm{C}_{15} \mathrm{H}_{13} \mathrm{~N}_{3}[\mathrm{MH}]^{+}$calcd: 236.1188 , found: 236.1185 .

1-Benzyl-4-(phthalimido-2-yl-methyl)-1 H-1,2,3-triazole (entry 2, Table 2). CAS No. 478555-31-6; 97\% yield as a white solid: $\mathrm{mp} 178-179{ }^{\circ} \mathrm{C} ;{ }^{1} \mathrm{H} \mathrm{NMR}\left(\mathrm{CDCl}_{3}, 200 \mathrm{MHz}\right) \delta 7.86-7.82$ (m, 2H), 7.73-7.69 (m, 2H), 7.51 (s, 1H), 7.37-7.26 (m, 5H), 5.48 $(\mathrm{s}, 2 \mathrm{H}), 4.97(\mathrm{~s}, 2 \mathrm{H}) ;{ }^{13} \mathrm{C} \mathrm{NMR}\left(\mathrm{CDCl}_{3}, 50 \mathrm{MHz}\right) \delta 167.6,143.1$, 134.4, 134.0, 132.0, 129.0, 128.7, 128.0, 123.4, 122.6, 54.1, 33.1. HRMS (FAB) $m / z \mathrm{C}_{18} \mathrm{H}_{14} \mathrm{O}_{2}[\mathrm{MH}]^{+}$calcd: 319.1195 , found: 319.1195 .

1-Benzyl-4-(2-phenylethyl)-1H-1,2,3-triazole (entry 3, Table 2). $99 \%$ yield as a bright yellow solid: mp $65{ }^{\circ} \mathrm{C} ;{ }^{1} \mathrm{H}$ NMR $\left(\mathrm{CDCl}_{3}, 400 \mathrm{MHz}\right) \delta$ 7.33-7.29 (m, 3H), 7.23-7.09 (m, 8H), $5.41(\mathrm{~s}, 2 \mathrm{H}), 2.96-2.94(\mathrm{~m}, 4 \mathrm{H}) ;{ }^{13} \mathrm{C} \mathrm{NMR}\left(\mathrm{CDCl}_{3}, 100 \mathrm{MHz}\right)$ $\delta 140.8,134.7,128.8,128.4,128.2,128.1,127.7,125.9,53.8$, 35.3, 27.4. HRMS (FAB) $m / z \mathrm{C}_{17} \mathrm{H}_{17} \mathrm{~N}_{3}[\mathrm{MH}]^{+}$calcd: 264.1501, found: 264.1499 .

1-Benzyl-4-[bis- $N, N$-( $t$-butyloxycarbonylmethyl)aminomethyl]-1 H-1,2,3-triazole (entry 4, Table 2). 97\% yield as a bright yellow solid: mp $59-60{ }^{\circ} \mathrm{C} ;{ }^{1} \mathrm{H} \mathrm{NMR}\left(\mathrm{CDCl}_{3}, 400 \mathrm{MHz}\right)$ $\delta 7.59$ (brs, $1 \mathrm{H}), 7.36-7.26(\mathrm{~m}, 5 \mathrm{H}), 5.51$ (s, 2H), 4.00 (brs, 2H), 3.45 (brs, $4 \mathrm{H}), 1.45(\mathrm{~s}, 18 \mathrm{H}) ;{ }^{13} \mathrm{C} \mathrm{NMR}\left(\mathrm{CDCl}_{3}, 100 \mathrm{MHz}\right) \delta$
170.3, 134.5, 128.8, 128.5, 128.2, 128.0, 80.9, 55.0, 54.1, 49.0, 28.0. HRMS (FAB) $m / z \mathrm{C}_{22} \mathrm{H}_{32} \mathrm{~N}_{4} \mathrm{O}_{4}[\mathrm{MH}]^{+}$calcd: 417.2502, found: 417.2504 .

$\mathrm{N}$-BOC-cis-4-(4-phenyl-1 H-1,2,3-triazol-1-yl)-L-proline methyl ester(entry 5, Table 2). 98\% yield as a white solid: mp $45-46{ }^{\circ} \mathrm{C} ;{ }^{1} \mathrm{H}$ NMR $\left(\mathrm{CDCl}_{3}, 200 \mathrm{MHz}\right) \delta 8.01(\mathrm{~s}, 1 \mathrm{H}), 7.83-7.77$ $(\mathrm{m}, 2 \mathrm{H}), 7.45-7.28(\mathrm{~m}, 3 \mathrm{H}), 5.23-5.17(\mathrm{t}, J=12.8 \mathrm{~Hz}, 1 \mathrm{H}), 4.48-$ $4.41(\mathrm{~m}, 1 \mathrm{H}), 4.18(\mathrm{dd}, J=11.4,7.4 \mathrm{~Hz}, 1 \mathrm{H}), 4.00-3.89(\mathrm{~m}, 1 \mathrm{H})$, 3.69 (s, 3H), 3.03-2.88 (m, 1H), 2.70-2.61 (m, 1H), 1.45 (s, 9H); ${ }^{13} \mathrm{C} \mathrm{NMR}\left(\mathrm{CDCl}_{3}, 50 \mathrm{MHz}\right) \delta 171.9,153.1,147.8,130.1,128.6$, 128.1, 125.5, 118.6, 80.7, 57.4, 52.1, 51.0, 35.9, 28.0. HRMS (FAB) $m / z \mathrm{C}_{19} \mathrm{H}_{24} \mathrm{~N}_{4} \mathrm{O}_{4}[\mathrm{MH}]^{+}$calcd: 373.1876 , found: 373.1877 .

N-BOC-cis-4-[4-(phthalimido-2-yl-methyl)-1H-1,2,3-triazol-1-yl]-L-proline methyl ester (entry 6, Table 2). 97\% yield as a white solid: $\mathrm{mp} 61-62{ }^{\circ} \mathrm{C} ;{ }^{1} \mathrm{H} \mathrm{NMR}\left(\mathrm{CDCl}_{3}, 200 \mathrm{MHz}\right) \delta$ 7.85-7.70 (m, 5H), 5.16 (s, 1H), $5.00(\mathrm{~s}, 2 \mathrm{H}), 4.46-4.39(\mathrm{~m}, 1 \mathrm{H})$, $4.17(\mathrm{dd}, J=11.4,7.4 \mathrm{~Hz}, 1 \mathrm{H}), 3.90-3.80(\mathrm{~m}, 1 \mathrm{H}), 3.70(\mathrm{~s}, 3 \mathrm{H})$, 3.02-2.87 (m, 1H), 2.67-2.57 (m, 1H), $1.44(\mathrm{~s}, 9 \mathrm{H}) ;{ }^{13} \mathrm{C} \mathrm{NMR}$ $\left(\mathrm{CDCl}_{3}, 50 \mathrm{MHz}\right) \delta 171.8,167.3,153.5,152.9,133.9,132.6$, 131.8, 123.2, 80.7, 57.5, 52.1, 50.8, 35.8, 35.0, 32.8, 27.9. HRMS (FAB) $m / z \mathrm{C}_{22} \mathrm{H}_{25} \mathrm{~N}_{5} \mathrm{O}_{6}[\mathrm{MH}]^{+}$calcd: 456.1883 , found: 456.1887.

N-BOC-cis-4-[4-(2-phenylethyl)-1H-1,2,3-triazol-1-yl]-Lproline methyl ester (entry 7, Table 2). $99 \%$ yield as a white solid: $\mathrm{mp} 82-83{ }^{\circ} \mathrm{C} ;{ }^{1} \mathrm{H} \mathrm{NMR}\left(\mathrm{CDCl}_{3}, 200 \mathrm{MHz}\right) \delta$ 7.32-7.15 $(\mathrm{m}, 6 \mathrm{H}), 5.10-5.03(\mathrm{~m}, 1 \mathrm{H}), 4.44-4.37(\mathrm{~m}, 1 \mathrm{H}), 4.12(\mathrm{dd}, J=$ $11.4,7.4 \mathrm{~Hz}, 1 \mathrm{H}), 3.82-3.73$ (m, 1H), 3.69 (s, 3H), 3.05-2.81 $(\mathrm{m}, 5 \mathrm{H}), 2.66-2.42(\mathrm{~m}, 1 \mathrm{H}), 1.43(\mathrm{~s}, 9 \mathrm{H}) ;{ }^{13} \mathrm{C} \mathrm{NMR}\left(\mathrm{CDCl}_{3}, 50\right.$ MHz) $\delta 172.0,153.2,140.9,128.3,128.2,126.0,80.8,57,5$, 52.2, 51.0, 35.9, 35.3, 28.1, 27.4. HRMS (FAB) $m / z \mathrm{C}_{21} \mathrm{H}_{28} \mathrm{~N}_{4} \mathrm{O}_{4}$ $[\mathrm{MH}]^{+}$calcd: 401.2189 , found: 401.2185 .

1-(2,3,4,6-Tetra- $O$-acetyl- $\beta$-D-glucopyranosyl)-4-phenyl$\mathbf{1 H}$-1,2,3-triazole (entry 8, Table 2). $94 \%$ yield as a white solid: mp $124-125{ }^{\circ} \mathrm{C} ;{ }^{1} \mathrm{H} \mathrm{NMR}\left(\mathrm{CDCl}_{3}, 400 \mathrm{MHz}\right) \delta 8.02(\mathrm{~s}, 1 \mathrm{H})$, 7.85-7.83 (m, 2H), 7.45-7.42 (m, 2H), 7.38-7.33 (m, 1H), 5.95 $(\mathrm{d}, J=9.6 \mathrm{~Hz}, 1 \mathrm{H}), 5.54(\mathrm{t}, J=9.6 \mathrm{~Hz}, 1 \mathrm{H}), 5.45(\mathrm{t}, J=9.6 \mathrm{~Hz}$, $1 \mathrm{H}), 5.28(\mathrm{t}, J=9.2 \mathrm{~Hz}, 1 \mathrm{H}), 4.34(\mathrm{dd}, J=12.4,4.8 \mathrm{~Hz}, 1 \mathrm{H})$, 4.17 (dd, $J=12.8,2.0 \mathrm{~Hz}, 1 \mathrm{H}), 4.05$ (ddd, $J=10.0,4.8,2.0 \mathrm{~Hz}$, 1H), 2.09 (s, 3H), $2.08(\mathrm{~s}, 3 \mathrm{H}), 2.04(\mathrm{~s}, 3 \mathrm{H}), 1.89(\mathrm{~s}, 3 \mathrm{H}) ;{ }^{13} \mathrm{C}$ $\mathrm{NMR}\left(\mathrm{CDCl}_{3}, 100 \mathrm{MHz}\right) \delta 170.4,169.9,169.3,168.9,148.5$, 129.8, 128.8, 128.5, 125.9, 117.7, 85.7, 75.1, 72.7, 70.2, 67.7, $61.5,20.6,20.5,20.47,20.1$. HRMS (FAB) $m / z \mathrm{C}_{22} \mathrm{H}_{25} \mathrm{~N}_{3} \mathrm{O}_{9}$ $[\mathrm{MH}]^{+}$calcd: 476.1669 , found: 476.1665 .

1-(2,3,4,6-Tetra- $O$-acetyl- $\beta$-D-glucopyranosyl)-4-(phthalimido-2-yl-methyl)-1H-1,2,3-triazole (entry 9, Table 2). 95\% yield as a white solid: $\operatorname{mp} 203-204{ }^{\circ} \mathrm{C} ;{ }^{1} \mathrm{H} \mathrm{NMR}\left(\mathrm{CDCl}_{3}, 400\right.$ $\mathrm{MHz}) \delta 7.93(\mathrm{~s}, 1 \mathrm{H}), 7.87-7.85(\mathrm{~m}, 2 \mathrm{H}), 7.74-7.72(\mathrm{~m}, 2 \mathrm{H})$, $5.94(\mathrm{~d}, J=8.8 \mathrm{~Hz}, 1 \mathrm{H}), 5.50-5.42(\mathrm{~m}, 2 \mathrm{H}), 5.26(\mathrm{t}, J=10.0$ $\mathrm{Hz}, 1 \mathrm{H}), 5.06-4.96$ (m, 2H), 4.30 (dd, $J=12.8,4.8 \mathrm{~Hz}, 1 \mathrm{H})$, 4.15 (dd, $J=12.8,2.4 \mathrm{~Hz}, 1 \mathrm{H}), 4.07$ (ddd, $J=10.4,4.8,2.4 \mathrm{~Hz}$, $1 \mathrm{H}), 2.06(\mathrm{~s}, 6 \mathrm{H}), 2.01(\mathrm{~s}, 3 \mathrm{H}), 1.82(\mathrm{~s}, 3 \mathrm{H}) ;{ }^{13} \mathrm{C} \mathrm{NMR}\left(\mathrm{CDCl}_{3}\right.$, $100 \mathrm{MHz}) \delta 170.3,169.7,169.1,168.6,167.3,143.2,133.9$, $131.8,123.2,121.4,85.3,74.8,72.4,70.0,67.5,61.4,32.7$, 20.4, 20.3, 20.3, 19.9. HRMS (FAB) $m / z \mathrm{C}_{25} \mathrm{H}_{26} \mathrm{~N}_{4} \mathrm{O}_{11}[\mathrm{MH}]^{+}$ calcd: 559.1676 , found: 559.1683 .

1-(2,3,4,6-Tetra- $O$-acetyl- $\beta$-D-glucopyranosyl)-4-(2-phenylethyl)-1H-1,2,3-triazole (entry 10, Table 2). 99\% yield as a white solid: mp $179-180{ }^{\circ} \mathrm{C} ;{ }^{1} \mathrm{H} \mathrm{NMR}\left(\mathrm{CDCl}_{3}, 400 \mathrm{MHz}\right) \delta$ 
7.44 (s, 1H), 7.30-7.25 (m, 2H), 7.21-7.17 (m, 3H), 5.87 (dd, $J=6.4,2.4 \mathrm{~Hz}, 1 \mathrm{H}), 5.42(\mathrm{dd}, J=6.8,2.8 \mathrm{~Hz}, 2 \mathrm{H}), 5.24$ (ddd, $J=10.0,6.4,2.8 \mathrm{~Hz}, 1 \mathrm{H}), 4.30$ (dd, $J=12.4,4.8 \mathrm{~Hz}, 1 \mathrm{H}), 4.14$ (dd, $J=12.8,2.4 \mathrm{~Hz}, 1 \mathrm{H}), 4.01$ (ddd, $J=10.0,5.2,2.4 \mathrm{~Hz}, 1 \mathrm{H}$ ), 3.07-2.97 (m, 4H), 2.07 (s, 3H), 2.06 (s, 3H), 2.02 (s, 3H), 1.85 $(\mathrm{s}, 3 \mathrm{H}) ;{ }^{13} \mathrm{C} \mathrm{NMR}\left(\mathrm{CDCl}_{3}, 100 \mathrm{MHz}\right) \delta 170.2,169.7,169.2$, 168.6, 147.8, 140.7, 128.2, 125.9, 119.0, 85.3, 74.7, 72.5, 70.0, 67.5, 61.4, 35.0, 27.2, 20.5, 20.3, 20.3, 19.9. HRMS (FAB) $\mathrm{m} / \mathrm{z}$ $\mathrm{C}_{24} \mathrm{H}_{29} \mathrm{~N}_{3} \mathrm{O}_{9}[\mathrm{MH}]^{+}$calcd: 504.1982 , found: 504.1981 .

1-(2,3,4,6-Tetra- $O$-acetyl- $\beta$-D-glucopyranosyl)-4-[bis- $N, N$ (t-butyloxycarbonylmethyl) aminomethyl]-1H-1,2,3-triazole (entry 11, Table 2). 93\% yield as a bright yellow solid: mp 130 $131{ }^{\circ} \mathrm{C} ;{ }^{1} \mathrm{H} \mathrm{NMR}\left(\mathrm{CDCl}_{3}, 400 \mathrm{MHz}\right) \delta 7.88(\mathrm{~s}, 1 \mathrm{H}), 5.92(\mathrm{~d}, J=$ $8.8 \mathrm{~Hz}, 1 \mathrm{H}), 5.48-5.40(\mathrm{~m}, 2 \mathrm{H}), 5.25$ (t, $J=9.6 \mathrm{~Hz}, 1 \mathrm{H}), 4.32$ (dd, $J=12.8,4.8 \mathrm{~Hz}, 1 \mathrm{H}), 4.15(\mathrm{dd}, J=12.4,2.0 \mathrm{~Hz}, 1 \mathrm{H})$, 4.09-4.06 (m, 3H), 3.44 (s, 4H), 2.09 (s, 3H), 2.07 (s, 3H), 2.03 $(\mathrm{s}, 3 \mathrm{H}), 1.87(\mathrm{~s}, 3 \mathrm{H}), 1.47(\mathrm{~s}, 18 \mathrm{H}) ;{ }^{13} \mathrm{CNMR}\left(\mathrm{CDCl}_{3}, 100 \mathrm{MHz}\right)$ $\delta 170.2,170.0,169.6,169.0,168.4,146.0,121.3,85.4,80.7$, 74.6, 72.3, 70.2, 67.5, 61.3, 54.9, 48.5, 27.9, 20.4, 20.24, 20.21, 19.8. HRMS (FAB) $m / z \mathrm{C}_{29} \mathrm{H}_{44} \mathrm{~N}_{4} \mathrm{O}_{13}[\mathrm{MH}]^{+}$calcd: 657.2983, found: 657.2986 .

4-Phenyl-1-[2-(phthalimido-2-yl)ethyl]-1 H-1,2,3-triazole (entry 12, Table 2). 97\% yield as a bright yellow solid: mp 157 $158{ }^{\circ} \mathrm{C} ;{ }^{1} \mathrm{H} \mathrm{NMR}\left(\mathrm{CDCl}_{3}, 400 \mathrm{MHz}\right) \delta 7.87(\mathrm{~s}, 1 \mathrm{H}), 7.80-7.77$ (m, 4H), 7.69-7.67 (m, 2H), 7.40-7.36 (m, 2H), 7.32-7.28 (m, $1 \mathrm{H}), 4.73(\mathrm{t}, J=6.4 \mathrm{~Hz}, 2 \mathrm{H}), 4.20(\mathrm{t}, J=6.4 \mathrm{~Hz}, 2 \mathrm{H}) ;{ }^{13} \mathrm{C} \mathrm{NMR}$ $\left(\mathrm{CDCl}_{3}, 100 \mathrm{MHz}\right) \delta 167.6,147.9,134.2,131.6,130.4,128.7$, 128.1, 125.7, 119.9, 47.8, 37.6. HRMS (FAB) $m / z \mathrm{C}_{18} \mathrm{H}_{14} \mathrm{~N}_{4} \mathrm{O}_{2}$ $[\mathrm{MH}]^{+}$calcd: 319.1195 , found: 319.1190 .

4-[Bis- $N, N$-(t-butyloxycarbonylmethyl)aminomethyl]-1-[2(phthalimido-2-yl)ethyl]-1 H-1,2,3-triazole (entry 13, Table 2). 97\% yield as a pale yellow solid: mp $131-132{ }^{\circ} \mathrm{C} ;{ }^{1} \mathrm{H}$ NMR $\left(\mathrm{CDCl}_{3}, 400 \mathrm{MHz}\right) \delta 7.82-7.71(\mathrm{~m}, 5 \mathrm{H}), 4.69$ (brs, 2H), 4.16 (brs, 2H), 4.01 (brs, 2H), 3.42 (brs, 4H), 1.46 (brs, $18 \mathrm{H}) ;{ }^{13} \mathrm{C}$ $\mathrm{NMR}\left(\mathrm{CDCl}_{3}, 100 \mathrm{MHz}\right) \delta 170.1,167.3,145.6,134.0,131.4$, 123.2, 80.8, 54.9, 48.6, 47.7, 37.5, 27.9. HRMS (FAB) $\mathrm{m} / \mathrm{z}$ $\mathrm{C}_{25} \mathrm{H}_{33} \mathrm{~N}_{5} \mathrm{O}_{6}[\mathrm{MH}]^{+}$calcd: 500.2509, found: 500.2513.

\section{Results and Discussion}

Primarily, polymeric ammonium salts having azide or alkyne functionality were designed as scavenger resins instead of a neutral polymer because of their better swelling properties in polar reaction media, in which the click reaction is usually carried out. These resins were prepared by quaternization of high chloromethyl-loaded $(1.80 \mathrm{mmol} / \mathrm{g})$ Merrifield resin with

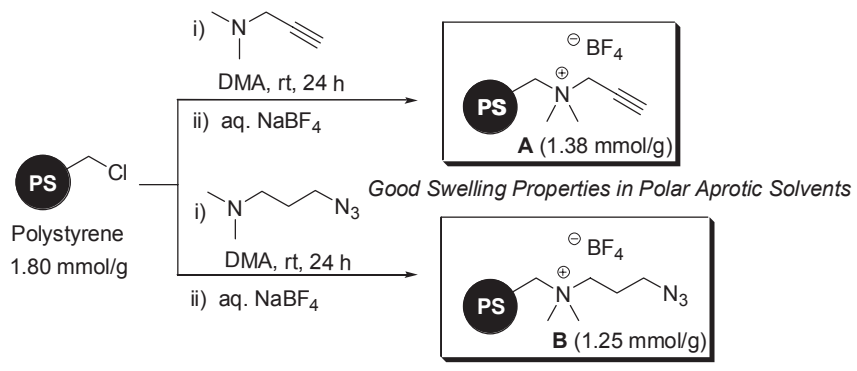

Scheme 1. Preparation of scavenger resins A and $\mathbf{B}$ excess amine having acetylene or azide functional group in dimethylacetamide (DMA) solvent at room temperature for $24 \mathrm{~h}$, followed by anion exchange with aqueous sodium tetrafluoroborate $\left(\mathrm{NaBF}_{4}\right)$.

The ammonium $\mathrm{BF}_{4}$ resin was chosen due to its better swelling property in polar aprotic solvent compared to ammonium $\mathrm{Cl}$ resin. ${ }^{12}$ Consequently, the resins $\mathbf{A}$ and $\mathbf{B}$ were obtained in $1.38 \mathrm{mmol} / \mathrm{g}$ for $\mathbf{A}$ and $1.25 \mathrm{mmol} / \mathrm{g}$ for $\mathbf{B}$ as determined by elemental analysis (calculated by nitrogen content) (Scheme 1). Both resins showed good swelling properties in several polar solvents such as acetonitrile, DMF, and DMSO over 5 fold swollen compared to dry resins.

To find out a suitable condition for both solution- and solidphase click reactions, we optimized the reaction condition using a simple click reaction of benzyl azide (1) and phenyl acetylene (2) (Table 1). The catalyst, base, and reaction solvent were varied for fast reaction completion, high regioselectivity, reducing byproducts such as oxidatively coupled acetylene dimer, ${ }^{13}$ and facile removal of other residues. Firstly, the Sharpless protocol, $10 \mathrm{~mol} \%$ of $\mathrm{CuSO}_{4} \cdot 5 \mathrm{H}_{2} \mathrm{O}$ (aqueous $0.2 \mathrm{M}$ )/Na-ascorbate (aqueous $0.2 \mathrm{M}$ ), was tried in DMF solvent (Table 1, entry 1). The reaction was completed within $1 \mathrm{~h}$, but it was difficult to remove the DMF solvent after the reaction. Thus, the same reaction was performed in more volatile acetonitrile solvent (entry 2). However, the reaction proceeded very slowly. On the next attempt, the Meldal protocol, $\mathrm{CuI} /$ triethylamine $\left(\mathrm{Et}_{3} \mathrm{~N}\right)$ was tested in acetonitrile solvent, and the reaction was completed within $3 \mathrm{~h}$. Problematically, an oxidatively coupled acetylene dimer and a 1,5-disubstituted triazole regioisomer were also formed (entry 3 ). Therefore, the catalytic system was returned back to the Sharpless condition, similar to entry 2 in Table 1. Although the addition of $\mathrm{Et}_{3} \mathrm{~N}$ accelerated the reaction, it remained slow (entry 4). As shown in entry 5, the efficiency of the reaction was improved when $10 \mathrm{~mol} \%$ of $\mathrm{CuSO}_{4} \cdot 5 \mathrm{H}_{2} \mathrm{O}$ (aqueous $0.2 \mathrm{M}$ )/Cu power was used in the presence of $\mathrm{Et}_{3} \mathrm{~N}$, affording fast reaction completion within $3 \mathrm{~h}$ with no byproducts. Furthermore, $5 \mathrm{~mol} \%$ of catalyst allowed the reaction to be completed within $6 \mathrm{~h}$ (entry 6). Consequently, $5 \mathrm{~mol} \%$ of $\mathrm{CuSO}_{4}$

Table 1. Optimization of solution-phase click reaction ${ }^{a}$

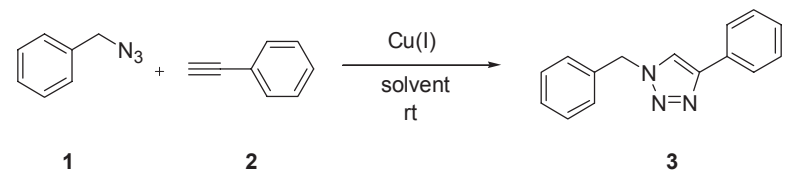

\begin{tabular}{cccrc}
\hline entry & $\mathrm{Cu}(\mathrm{I})^{b}$ & solvent & time & comments \\
\hline $\mathbf{1}$ & $\mathrm{A}$ & $\mathrm{DMF}$ & $1 \mathrm{~h}$ & non volatile DMF \\
$\mathbf{2}$ & $\mathrm{A}$ & $\mathrm{CH}_{3} \mathrm{CN}$ & $3 \mathrm{~d}$ & slow \\
$\mathbf{3}$ & $\mathrm{B}$ & $\mathrm{CH}_{3} \mathrm{CN}$ & $3 \mathrm{~h}$ & regioisomer \& acetylene dimer \\
$\mathbf{4}$ & $\mathrm{C}$ & $\mathrm{CH}_{3} \mathrm{CN}$ & $>24 \mathrm{~h}$ & slow \\
$\mathbf{5}$ & $\mathrm{D}$ & $\mathrm{CH}_{3} \mathrm{CN}$ & $3 \mathrm{~h}$ & \\
$\mathbf{6}$ & $\mathrm{E}$ & $\mathrm{CH}_{3} \mathrm{CN}$ & $6 \mathrm{~h}$ & \\
\hline
\end{tabular}

${ }^{a} \mathrm{All}$ reactions were carried out on a $0.5 \mathrm{mmol}$ of $\mathbf{1}$ and 1.1 equiv of $2 .{ }^{b} \mathrm{~A}$ : $10 \mathrm{~mol} \% \mathrm{CuSO}_{4} \cdot 5 \mathrm{H}_{2} \mathrm{O}, 10 \mathrm{~mol} \% \mathrm{Na}$-ascorbate. B: $10 \mathrm{~mol} \% \mathrm{CuI}, 10$ mol $\% \mathrm{Et}_{3} \mathrm{~N}$. C: $10 \mathrm{~mol}^{\%} \mathrm{CuSO}_{4} \cdot 5 \mathrm{H}_{2} \mathrm{O}, 10 \mathrm{~mol} \%$ Na-ascorbate, $10 \mathrm{~mol} \%$ Et 3 N. D: 10 mol $\% \mathrm{CuSO}_{4} \cdot 5 \mathrm{H}_{2} \mathrm{O}, 10 \mathrm{~mol} \% \mathrm{Cu}, 10 \mathrm{~mol} \% \mathrm{Et}_{3} \mathrm{~N}$. E: $5 \mathrm{~mol} \%$ $\mathrm{CuSO}_{4} \cdot 5 \mathrm{H}_{2} \mathrm{O}, 5 \mathrm{~mol} \% \mathrm{Cu}, 5 \mathrm{~mol} \% \mathrm{Et}_{3} \mathrm{~N}$. 


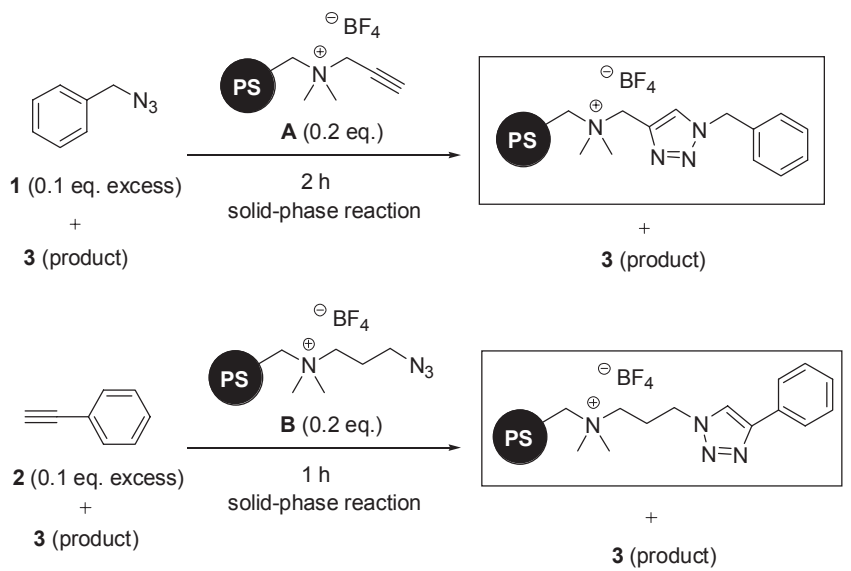

Scheme 2. Heterogeneous click reactions

(aqueous $0.2 \mathrm{M}$ )/Cu/ $/ \mathrm{Et}_{3} \mathrm{~N}$ in acetonitrile solvent was chosen as the optimal condition for the solution-phase click reaction.

After optimization of the solution-phase click reaction, the heterogeneous click reaction was investigated by adding corresponding acetylene or azide resin (A or $\mathbf{B})$ to the reaction mixture. The reaction was completed within $2 \mathrm{~h}$ and $1 \mathrm{~h}$, respectively, in which 0.2 equiv of scavenger resin $\mathbf{A}$ or $\mathbf{B}$ was used for complete scavenging of 0.1 equiv of unreacted starting molecule azide (1) or acetylene (2), respectively (Scheme 2).

After the solid-phase click reaction, we attempted to remove impurities such as copper species, $\mathrm{Et}_{3} \mathrm{~N}$, and acetonitrile solvent that remained in the reaction mixture. Recently, our group reported that $\mathrm{CuI}$ can be immobilized onto polystyrene-based ammonium salt resin for heterogeneous solid catalyst. ${ }^{10}$ Soluble copper residue could be consistently immobilized onto scaven-

(a)

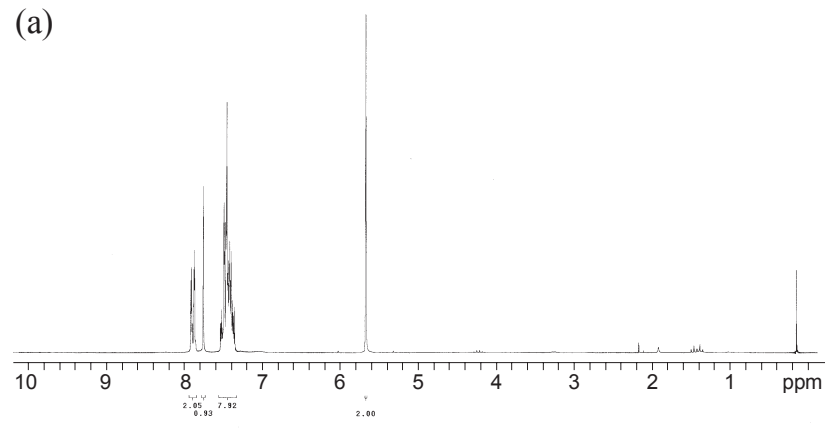

(b)

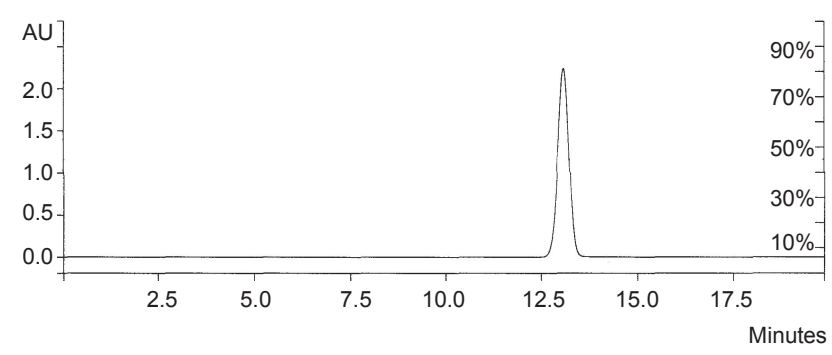

Figure 1. Analysis of crude compound 3. (a) ${ }^{1} \mathrm{H}$ NMR, (b) HPLC profile: conditions: $\mathrm{C}-18(250 \times 4.6 \mathrm{~mm})$, acetonitrile/ $\mathrm{H}_{2} \mathrm{O}(45 / 55)$, at $254 \mathrm{~nm}$ and $1 \mathrm{~mL} / \mathrm{min}$.
Table 2. Various click reactions ${ }^{a}$

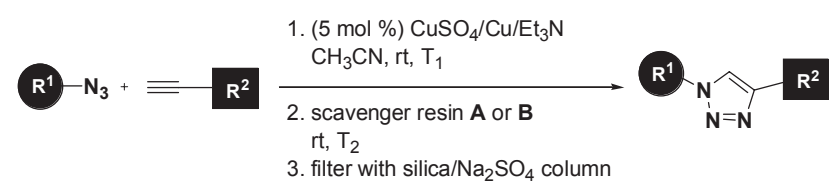

entry azide/acetylene acetylene/azide $\operatorname{resin} \mathrm{T}_{1} / \mathrm{T}_{2}$ yield purity

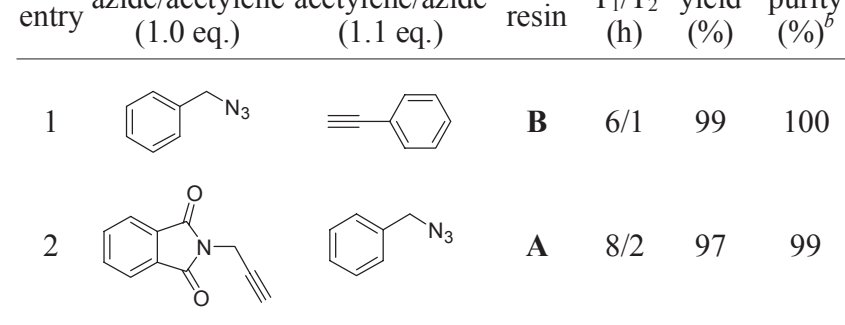

3

N

4<smiles>C#CCN(CC(=O)OC(C)(C)C)OC(=O)CO</smiles>

5

$\mathrm{N}_{3}$

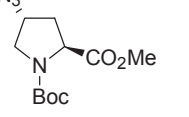<smiles>C#Cc1ccccc1</smiles>

B $\quad 18 / 1 \quad 98$

100

6

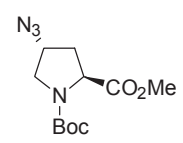<smiles>C#CCN1C(=O)c2ccccc2C1=O</smiles>

B $\quad 8 / 3 \quad 97$

$\mathrm{ND}^{c}$

7

$\mathrm{N}_{3}$

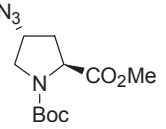<smiles>C#CCCc1ccccc1</smiles>

B $10 / 2 \quad 99$

98

8

AcO<smiles>C#Cc1ccccc1</smiles>

B $\quad 8 / 1 \quad 94$

100

9

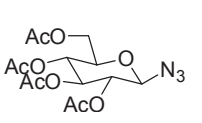<smiles>C#CCN1C(=O)c2ccccc2C1=O</smiles>

B $8 / 3 \quad 95$

$\mathrm{ND}^{c}$

10

$\mathrm{N}_{3}$<smiles>C#CCCc1ccccc1</smiles>

B $10 / 2 \quad 99$

100

11<smiles>C#CCN(CC(=O)OC(C)(C)C)CC(=O)OC(C)(C)C</smiles>

B $\quad 8 / 5 \quad 93 \quad \mathrm{ND}^{c}$

12

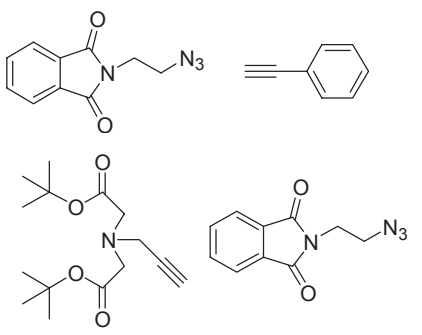

$8 / 1 \quad 97$

96

A $\quad 6 / 2 \quad 97$

98

$\overline{{ }^{a} \text { All reactions were carried out using } 0.5 \mathrm{mmol} \text { of acetylene or azide, and }}$ 1.1 eq. of counterpart azide or acetylene. After complete solution phase reaction, corresponding resin ( 0.2 eq.) $\mathbf{A}$ or $\mathbf{B}$ was added. ${ }^{b}$ Determined by ${ }^{1} \mathrm{H}-\mathrm{NMR}$ and confirmed by HPLC. ${ }^{C}$ Not determined. 
ger resins and removed by filtration, together with insoluble copper metal.

However, a tiny amount of copper still remained in the filtrate solution. This was completely removed by eluting the reaction mixture through a short column packed with $\mathrm{Na}_{2} \mathrm{SO}_{4}$ and silica gel, in which the $\mathrm{Na}_{2} \mathrm{SO}_{4}$ was required to remove the small amount of water from the aqueous $\mathrm{CuSO}_{4}$ solution used. The column was washed with additional ethyl acetate $(5 \mathrm{~mL} \times 2)$.

This filtration has proved to be sufficient to remove all copper species by ICP-MS (inductively coupled plasma - mass spectroscopy) analysis to detect $\mathrm{Cu}$ metal in the final solution. The analysis sample was prepared in $10 \mathrm{~mL}$ acidic aqueous solution (calculated, 157.5 ppm; observed, $0.149 \mathrm{ppm} ; \rightarrow 0.095 \%$ ). The resulting acetonitrile solution obtained after filtration was evaporated to remove the acetonitrile and $\mathrm{Et}_{3} \mathrm{~N}$ and thereby afford a quantitatively yielded crude product. ${ }^{1} \mathrm{H}$ NMR spectrum (Figure 1a) and HPLC analysis (Figure 1b) confirmed the high purity of almost $100 \%$ of the crude product 3 .

Using the aforementioned optimized reaction/ purification condition, the quick preparation of click product was further explored with several substrates in the one-pot reaction of solution-phase and subsequent solid-phase (Table 2). All reactions using $5 \mathrm{~mol} \%$ of each $\mathrm{CuSO}_{4}, \mathrm{Cu}$, and $\mathrm{Et}_{3} \mathrm{~N}$ in $\mathrm{CH}_{3} \mathrm{CN}$ at room temperature afforded corresponding high regioselective products with excellent yield and high purity. While the first solution-phase reaction required a relatively longer reaction time, the solid-phase scavenging step took less time. After each reaction was completed, the reaction mixture was diluted with ethyl acetate and filtered with the $\mathrm{Na}_{2} \mathrm{SO}_{4} /$ silica column to remove the remaining $\mathrm{Cu}$ metal and water. The triethylamine base was removed by evaporation under reduced pressure. As shown in Table 2, all desired regioselective 1,4-disubstituted 1,2,3-triazoles were uneventfully obtained in high yield and excellent purity.

In conclusion, a new technique for rapid purification of the click reaction was developed using azide/acetylene scavenger resins. These resins were prepared by quaternization of high chloromethyl-loaded, Merrifield resin with azide/acetylene amine. The corresponding ammonium salt resins showed excellent swelling properties in polar aprotic solvents such as acetonitrile, DMF and DMSO. The copper metal and water residue that remained after the reaction were removed by using $\mathrm{Na}_{2} \mathrm{SO}_{4} /$ silica cartridge column. The optimal condition, including reaction and purification, was applied to 13 additional click reactions, giving high yield and excellent purity. We expect this method to become the general procedure for the high throughput synthesis of 1,4-disubstituted 1,2,3-triazoles without conventional purification.

Acknowledgments. This work was supported by the Converging Research Program (2009-0081956) and the Real-Time Molecular Imaging Project (2009-0065588) through the Na- tional Research Foundation (NRF) of Korea funded by the Ministry of Education, Science and Technology and Korea Health 21 R\&D Project, Ministry of Health \& Welfare (A062254).

Supporting Information. ${ }^{1} \mathrm{H}$ and ${ }^{13} \mathrm{C}$ NMR spectra and HPLC chromatograms are available on request from the correspondence authors. Fax: +82-2-715-2411 (DYC); E-mail: bslee@ futurechem.co.kr (BSL); dychi@sogang.ac.kr (DYC).

\section{References}

1. Tornoe, C. W.; Christensen, C.; Meldal, M. J. Org. Chem. 2002, $67,3057$.

2. (a) Kolb, H. C.; Finn, M. G.; Sharpless, K. B. Angew. Chem. Int. Ed. 2001, 40, 2004. (b) Rostovtsev, V. V.; Green, L. G.; Fokin, V. V.; Sharpless, K. B. Angew. Chem. Int. Ed. 2002, 41, 2596.

3. for small molecule synthesis: (a) Ng, S. L.; Yang, P.-Y.; Chen, K. Y.-T.; Srinivasan, R.; Yao, S. Q. Org. Biomol. Chem. 2008, 6, 844. (b) Chen, J.; Nikolovska-Coleska, Z.; Yang, C. Y.; Gomez, C.; Gao, W.; Krajewski, K.; Jiang, S.; Roller, P.; Wang, S. Bioorg. Med. Chem. Lett. 2007, 17, 3939.

4. for bioconjugation: (a) Geng, J.; Mantovani, G.; Tao, L.; Nicolas, J.; Chen, G.; Wallis, R.; Mitchell, D. A.; Johnson, B. R. G.; Evans, S. D.; Haddleton, D. M. J. Am. Chem. Soc. 2007, 129, 15156. (b) Wang, Q.; Chan, T. R.; Hilgraf, R.; Fokin, V. V.; Sharpless, K. B.; Finn, M. G. J. Am. Chem. Soc. 2003, 125, 3192.

5. for polymerization: (a) Qin, A.; Jim, C. K. W.; Lu, W.; Lam, J. W. Y.; Häussler, M.; Dong, Y.; Sung, H. H. Y.; Williams, I. D.; Wong, G. K. L.; Tang, B. Z. Macromolecules 2007, 40, 2308. (b) Lee, J. W.; Han, S. C.; Kim, J. H.; Ko, Y. H.; Kim, K. Bull. Korean Chem. Soc. 2007, 28, 1837. (c) Lee, J. W.; Kim, B.-K.; Jin, S.-H. Bull. Korean Chem. Soc. 2005, 26, 833. (d) Wu, P.; Feldman, A. K.; Nugent, A. K.; Hawker, C. J.; Scheel, A.; Voit, B.; Pyun, J.; Fréchet, J. M. J.; Sharpless, K. B.; Fokin, V. V. Angew. Chem. Int. Ed. 2004, 43, 3928.

6. for radiolableling: (a) Li, Z.-B.; Wu, Z.; Chen, K.; Chin, F. T.; Chen, X. Bioconjugate Chem. 2007, 18, 1987. (b) Sirion, U.; Kim, H. J.; Lee, J. H.; Seo, J. W.; Lee, B. S.; Lee, S. J.; Oh, S. J.; Chi, D. Y. Tetrahedron Lett. 2007, 48, 3953.

7. (a) Kolb, H. C.; Sharpless, K. B. Drug Discovery Today 2003, 8 , 1128. (b) Tron, G. C.; Pirali, T.; Billington, R. A.; Canonico, P. L.; Sorba, G.; Genazzani, A. A. Medicinal Research Reviews 2008, $28,278$.

8. (a) Brik, A.; Alexandratos, J.; Lin, Y. C.; Elder, J. H.; Olson, A. J.; Wlodawer, A.; Goodsell, D. S.; Wong C. H. ChemBioChem 2005, 6, 1167. (b) Angell, Y.; Burgess, K. J. Org. Chem. 2005, 70, 9595. (c) Bock, V. D.; Speijer, D.; Hiemstra, H.; van Maarseveen, J. H. Org. Biomol. Chem. 2007, 5, 971. (d) Hitotsuyanagi, Y.; Motegi, S.; Hasuda, T.; Takeya, K. Org. Lett. 2004, 6, 1111. (e) Horne, W. S.; Stout, C. D.; Ghadiri, M. R. J. Am. Chem. Soc. 2003, 125, 9372. (f) Horne, W. S.; Yadav, M. K.; Stout, C. D.; Ghadiri, M. R. J. Am. Chem. Soc. 2004, 126, 15366.

9. Girard, C.; Önen, E.; Aufort, M.; Beauvière, S.; Samson, E.; Herscovici, J. Org. Lett. 2006, 8, 1689.

10. Sirion, U.; Bae, Y. J.; Lee, B. S.; Chi, D. Y. Synlett 2008, 2326.

11. Smith, C. D.; Baxendale, I. R.; Lanners, S.; Hayward, J. J.; Smith, S. C.; Ley, S. V. Org. Biomol. Chem. 2007, 5, 1559.

12. Lee, B. S.; Mahajan, S.; Janda, K. D. Tetrahderon Lett. 2005, 46, 807.

13. Tregre, G. J.; Mathias, L. J. J. Polym. Sci. Pol. Chem. 1997, 35, 587. 